\title{
Efficacy of a Single Intra-Articular HYMOVIS ONE Injection for Managing Symptomatic Hip Osteoarthritis: A I2-Month Follow-Up Retrospective Analysis of the ANTIAGE Register Data
}

This article was published in the following Dove Press journal:

Orthopedic Research and Reviews

\begin{abstract}
Alberto Migliore ${ }^{1,2}$
Bruno Frediani ${ }^{3}$

Gianfranco Gigliucci (D)

Calogero Foti $\mathbb{D D}^{4}$

Sergio Crimaldi $\mathbb{D}^{5}$

Orazio De Lucia $\left(1 D^{6}\right.$

Giovanni lolascon (iD ${ }^{7}$

'Rheumatology Unit, S. Pietro FBF Hospital, Rome, Italy; ${ }^{2}$ Department of Internal Medicine, S. Pietro FBF Hospital, Rome, Italy; ${ }^{3}$ Medical and Surgical Science and Neuroscience Department, Rheumatology Section, University of Siena, O.U. of Osteo-Articular Diagnostic Procedures, Siena, Italy; ${ }^{4}$ Physical and Rehabilitation Medicine Department, University of Rome Tor Vergata, Rome, Italy; ${ }^{5}$ Orthopaedics and Traumatology Department, Lucca Hospital, USL Tuscany Northwest, Lucca, Italy; ${ }^{6}$ Division of Rheumatology, Gaetano Pini Institute, Milan, Italy; ${ }^{7}$ Physical and Rehabilitation Medicine Department, Napoli University, Naples, Italy
\end{abstract}

Correspondence: Alberto Migliore Department of Internal Medicine, S. Pietro FBF Hospital, Rome 00189 , Italy Tel +390497443620

Fax +390497443622

Email alberto.migliore.12@outlook.it
Purpose: The use of ultrasound (US) guidance has allowed hip osteoarthritis to be treated with intra-articular (IA) injections. HYMOVIS ONE (HYADD4-G) is a new hyaluronic acid (HA) derivative product with unusual characteristics, and it has been used with good results in knee osteoarthritis (OA). This study assessed the efficacy and safety of a single HYMOVIS ONE injection in patients affected by symptomatic hip OA.

Patients and Methods: This post-marketing cohort study assessed data from the ANTIAGE Register. Inclusion criteria were age $\geq 40$ years, symptomatic hip OA (Kellgren-Lawrence grade I-III) of $\geq 1$-year duration, and $\geq 12$ months follow-up. All patients received a single HYMOVIS ONE (32 mg/4 mL) injection at baseline. Values for $10-\mathrm{cm}$ visual analogue scale (VAS) pain scores, the Lequesne index, and nonsteroidal anti-inflammatory drug (NSAID) consumption were evaluated at 6 and 12 months. Adverse events were also recorded.

Results: The included patients $(n=198)$ consisted of $42.5 \%$ women, with a mean $( \pm$ SD) age at baseline of $62( \pm 14.2)$ years and a mean $( \pm$ SD) body mass index of $26.3( \pm 2.5)$. The mean (SD) Lequesne index and VAS pain scores at baseline were $11.5( \pm 4.6)$ and $6.4 \mathrm{~cm}$ $( \pm 2.2)$, respectively. All groups exhibited statistically significant reductions at all time points compared to baseline. At 12 months, the VAS pain score was reduced by $17.2 \%$, the Lequesne index by $33.7 \%$, and NSAID consumption by $41.7 \%$.

Conclusion: Our study supports the clinical efficacy and safety of a single HYMOVIS ONE injection for managing symptoms in patients with hip OA, confirming previous data on the use of HYMOVIS as a background therapy in the management of knee osteoarthritis.

Keywords: hip osteoarthritis, viscosupplementation, HYMOVIS ONE, HYADD4-G

\section{Introduction}

Hip osteoarthritis (OA) is one of the major causes of pain and disability in the western population. Pain consequent to hip OA may significantly hinder the patient's ability to perform daily activities, and disablement may be so impairing that it leads to social isolation. The disease prevalence is approximately $17 \%$ in white males and $9 \%$ in white women over 60 years of age. ${ }^{1}$ Treatment aims to relieve pain and to preserve or restore joint mobility in milder cases, and when the disease stage is more severe, to delay total hip replacement (THR) ${ }^{2}$ given the fact that THR, even if $90 \%$ effective, ${ }^{3,4}$ is associated with substantial adverse effects and relevant mortality rates. 5,6 
Pharmacological treatments include acetaminophen as a first option, followed by nonsteroidal anti-inflammatory drugs (NSAIDs) for non-responding patients, to be taken with a gastroprotective agent or a selective COX-2 inhibitor. When NSAIDs are ineffective, patients may be treated with opioid analgesics. ${ }^{7}$ Possibly, patients will take medications for their remaining lifespan, and long-term effects and the economic burden of medications should be the subject of careful consideration. ${ }^{8}$ Therefore, alternative options for managing hip OA symptoms are actively being investigated, as they may reduce analgesic consumption.

Among these, viscosupplementation (VS) is receiving increasing consideration as a valid option for managing symptoms of mild to moderate hip OA, given the results of recent randomized clinical trials ${ }^{2,9}$ and recommendations of expert consensuses. ${ }^{10,11}$ Hip VS is normally performed under ultrasound (US) or fluoroscopy guidance. This has replaced the use of anatomic landmarks, reducing morbidity and increasing precision in intra-articular (IA) delivery. ${ }^{12,13}$

Among viscosupplementation agents, hyaluronic acid (HA) has been extensively studied because it is a natural component of synovial fluid (SF). Here, its concentration varies between 0.5 and $4 \mathrm{mg} / \mathrm{mL}$ and its molecular weight (MW) ranges from 2 to $10 \mathrm{MDa} .{ }^{14,15}$ Under dynamic loading, weak non-ionic forces between HA molecules confer to HA unique non-Newtonian rheological properties, including shear thinning and reduction of viscosity, ${ }^{16-18}$ which confers outstanding visco-elasticity, shock absorption, and lubricating properties to the SF, particularly during high shear or compression conditions. ${ }^{19}$ Joint arthropathies, including $\mathrm{OA}$, are associated with a reduction of the molecular weight and concentration of hyaluronan in the synovial fluid, ${ }^{20}$ causing SF rheological properties to worsen, and therefore contributing further to OA progression. ${ }^{15}$

Novel injectable HA formulations often present a modified HA chain. Modifications are introduced to modulate the rheological properties and resorption kinetics of the formulation and increase the effectiveness in the clinical setting. ${ }^{21,22}$ A HA alkyl-derivative, non-chemically cross-linked-based formulation (HYADD 4-G, Fidia Farmaceutici, Abano Terme, Italy) has recently entered the market as a viscosupplementation agent. It is a highly viscoelastic injectable hydrogel whose peculiar rheological properties are derived from the presence of hexadecyl (C-16) hydrophobic chains that facilitate its aggregation even at low aqueous concentrations. ${ }^{23,24}$ Priano and colleagues $^{25}$ have shown that $24 \mathrm{mg} / 3 \mathrm{~mL}$ high molecular- weight viscoelastic hyaluronan (HYMOVIS/HYADD4-G) injections reduced Western Ontario and McMaster Universities Osteoarthritis (WOMAC) scores and NSAID/ acetaminophen consumption for at least 6 months in 698 patients with mild to severe knee OA (Kellgren-Lawrence grades II-IV) who underwent two consecutive infiltrations 1 week apart. In a subpopulation of 106 patients, efficacy against pain lasted approximately 12 months. ${ }^{25}$

Previously, Bisicchia et $\mathrm{al}^{26}$ conducted a single-center, single-blind, prospective randomized controlled clinical study with 1-year follow-up, and showed that $2(24 \mathrm{mg} /$ $3 \mathrm{~mL}$ ) HYMOVIS injections did not have significantly higher side effects when compared to corticosteroid injections and provided better short-term control of symptoms in patients with mild to moderate knee osteoarthritis. Our group has extensively studied the effect of HA viscosupplementation in managing symptoms of hip OA, using non-modified HA within the 1.5-2.0 MDa range injected under US guidance. ${ }^{27-32}$

In a recent prospective, post-market seven-year followup cohort study whose data were collected from the Associazione Nazionale per la Terepia Intra Articolare dell'Anca con Guida Ecografica (ANTIAGE) register, we found that hip OA patients who received non-modified HA injections at least every 6 months showed, at all time points and compared to baseline, a statistically significant improvement of Lequesne index, pain visual analogue scale (VAS), NSAID intake, and global medical and patient assessments. ${ }^{32}$ Modifications made to HA to improve its rheology and resorption kinetics may, in principle, modulate the clinical response of patients to IA injection; consequently, the optimal therapeutic protocol for a given HA formulation should be investigated on a case-by-case basis. As the ANTIAGE register contains records concerning hip OA patients who were treated by a single $(32 \mathrm{mg} / 4 \mathrm{~mL})$ HYMOVIS ONE IA injection, and as single-injection protocols may be of special clinical interest because of their intrinsic enhanced safety and patient acceptability, we decided to retrospectively analyze the records to assess the safety and performance profile of this modified injectable HA formulation, in relation to this administration regimen.

\section{Materials and Methods Study Design}

This is a multicentre, observational, retrospective analysis of patient register data regarding subjects affected by mild to moderate hip OA, who were treated with a single 
(32 mg/4 mL) HYMOVIS ONE IA injection and were followed up for at least 12 months at 6 Italian orthopaedic/rheumatology centres, namely the Rheumatology Unit at the S. Pietro FBF Hospital, Rome (the referral centre); the Osteo-Articular Diagnostic Procedures Operative Unit, at the Medical and Surgical Science and Neuroscience Department, Siena; the Physical and Rehabilitation Medicine Department at University of Rome Tor Vergata, Rome; the Orthopaedics and Traumatology Department at Lucca Hospital, Lucca; the Rheumatology Department at the Gaetano Pini Institute, Milan; and the Physical and Rehabilitation Medicine Department, Napoli University, Naples.

Records were retrieved from the ANTIAGE register, a prospectively built database holding deidentified patient data, which is described elsewhere. ${ }^{33}$ Data extraction and analysis were approved by the ethics committee of each centre.

\section{Study Population}

Records were included if related to patients (both males and females) who were $\geq 40$ years of age; had BMI $<30$; had an X-ray-confirmed diagnosis of mild to moderate, Kellgren and Lawrence ${ }^{34}$ (KL) grade I to III hip OA; showed moderate to severe pain, corresponding to 40 to $80 \mathrm{~mm}$ VAS scores at the affected hip; were treated with one (32 mg/4 mL) HYMOVIS ONE IA injection and followed up for at least 12 months.

Patients included in the study had their VAS pain scores, Lequesne index scores, and nonsteroidal antiinflammatory drug (NSAID) consumption assessed at baseline (before the IA injection) and 6 and 12 months thereafter. Records were excluded if patients showed any of the following: severe OA (KL grade IV); concomitant use of oral anticoagulant therapy, severe comorbidities (eg, rheumatologic disease, low back pain, femoral head osteonecrosis), significant varus or valgus deformity requiring surgical correction, inflammatory diseases that might affect joints, positive anamnesis for sepsis or subacute infection in any joint, relevant lymphatic stasis at the treated hip, hypersensitivities to any components of HAbased injection, or treatment with HA or HA derivatives in the previous 6 months or with IA steroids in the previous 3 months.

\section{Procedures}

Radiological evaluations according to the KL hip OA scale were made on a non-weight-bearing X-ray, and were taken no more than 6 months prior to the start treatment date. Pain was evaluated using a 100-mm VAS score.

IA injections were performed under US guidance ${ }^{12}$ according to an anterosuperior approach that has been perfected and standardized over time by the authors. ${ }^{13,33}$ The approach utilizes an US transducer together with a sterile bioptic target device while patients are placed supine with the hip at $15-20^{\circ}$ internal rotation, and the hip joint is scanned according to an anterior parasagittal approach, lateral to the femoral vessels. The transducer is aligned with the long axis of the femoral neck, comprising the acetabulum and the femoral head. The injection is performed by inserting a 20 -gauge $(9 \mathrm{~cm})$ spinal needle into the biopsy guide. Using biopsy real-time guidance software, the needle is advanced into the anterior capsular recess, at the level of the femoral head and, when in contact with it, is retracted by $1 \mathrm{~mm}$ before starting the injection. The injection into the articular space was verified by real-time monitoring (direct visualization of viscous fluid or air bubbles) and power Doppler imaging (flow signals in the intra-articular recess). The colour Doppler view also enabled the physician to avoid blood vessels.

\section{Objectives and Endpoints}

The primary study objective was to assess the duration of symptom relief and functional improvement following a single HYMOVIS ONE injection, when measured using the VAS pain and Lequesne index scores as well as NSAID consumption. The secondary study objective was to investigate the safety of the HYMOVIS ONE administration regimen, in terms of frequency and type of adverse events recorded.

\section{Statistical Analysis}

Record screening and inclusion was carried out at the single-centre level. Included records were then transferred to the referral centre (Rheumatology Unit, S. Pietro FBF Hospital). Here, they were re-checked according to the inclusion and exclusion criteria. The data were extracted from records, and homogeneity of patient distribution among different centres concerning age, gender, body mass index (BMI), and $\mathrm{KL}$ and $\mathrm{OA}$ scores at baseline was assessed using parametric or non-parametric analysis of variance tests, according to the results of normality checks. Normality of data was checked using ShapiroWilks tests. 
Patients demographics as well as adverse event type and frequency were described by means of descriptive statistics that is, absolute or relative frequencies (\%) for discrete variables and as mean \pm standard deviation (SD) for continuous ones, as they all were found to have a normal distribution. To investigate if treatment with a single HYMOVIS ONE injection had a statistically significant effect on the performance endpoints of interest, the VAS and Lequesne scores collected at each control visit were compared to those at baseline by means of $t$-tests for paired data. Statistical test results were regarded as significant when $\mathrm{p}<0.05$.

\section{Results}

Records were analysed for 198 patients (114 men and 84 women) with a mean age of $62 \pm 14.2$ years. Characteristics of the study cohort at baseline are shown in Table 1. Most patients $(90.9 \%)$ were afflicted with KL OA grade II or III and were on average overweight, with their BMI being $26.3 \pm 2.5$.

Patients experienced a significant reduction of all scores under consideration compared to baseline (Table 2). At 6 months, compared to baseline, VAS pain decreased by $15.6 \%$, and at 12 months, it was still reduced by a similar amount (17.2\%) (Figure 1). Both variations were statistically significant compared to baseline $(\mathrm{p}<0.05)$. The Lequesne index underwent a $19.0 \%$ reduction after 6 months, and at

Table I Characteristics of the Study Cohort at Baseline

\begin{tabular}{|l|l|}
\hline Patients (n) & 198 \\
Men & $114(57.5 \%)$ \\
Women & $84(42.5 \%)$ \\
Age, years, mean (SD) & $62( \pm 14.2)$ \\
BMI, mean (SD) & $26.3( \pm 2.5)$ \\
Weight, kg, mean (SD) & $73.7( \pm 10.6)$ \\
Height, cm, mean (SD) & $169.7( \pm 7.2)$ \\
Lequesne index, mean (SD) & $11.5( \pm 2.5)$ \\
Pain VAS, mean (SD) & $6.4( \pm 2.2)$ \\
NSAID intake (days/months mean, (SD)) & $16.3( \pm 4.6)$ \\
\hline Age classes & \\
<65 y.o. & $95(48.0 \%)$ \\
$>65$ y.o. & $103(52.0 \%)$ \\
\hline Hip affected & \\
Right & $85(42.9 \%)$ \\
Left & $74(37.4 \%)$ \\
Bilateral & $39(19.7 \%)$ \\
\hline Kellgren-Lawrence radiological index & \\
Grade I & $18(9.1 \%)$ \\
Grade II & $79(39.9 \%)$ \\
Grade III & $101(51.0 \%)$ \\
\hline
\end{tabular}

Table 2 Pain VAS, Lequesne Index, and NSAID Consumption Variation Over Time

\begin{tabular}{|l|l|l|l|l|l|}
\hline & Baseline & 6 Months & $\%$ & $\begin{array}{l}12 \\
\text { Months }\end{array}$ & $\%$ \\
\hline Pain VAS & $6.4 \pm 2.2$ & $\begin{array}{l}5.4 \pm 2.0^{*} \\
(\mathrm{p}=0.002)\end{array}$ & -15.6 & $\begin{array}{l}5.3 \pm 1.9^{*} \\
(\mathrm{p}=0.004)\end{array}$ & -17.2 \\
\hline $\begin{array}{l}\text { Lequesne } \\
\text { index }\end{array}$ & $11.5 \pm 4.6$ & $\begin{array}{l}9.3 \pm 3.7^{*} \\
(\mathrm{p}=0.012)\end{array}$ & -19.0 & $\begin{array}{l}7.6 \pm 2.4^{*} \\
(\mathrm{p}=0.008)\end{array}$ & -33.7 \\
\hline $\begin{array}{l}\text { NSAIDs } \\
\text { consumption } \\
\text { (days/month) }\end{array}$ & $16.3 \pm 6.0$ & $\begin{array}{l}12.8 \pm 3.2^{*} \\
(\mathrm{p}=0.007)\end{array}$ & -21.8 & $\begin{array}{l}9.5 \pm 2.1 * \\
(\mathrm{p}=0.009)\end{array}$ & -41.7 \\
\hline
\end{tabular}

Notes: All values are given as the mean \pm SD. *Significant difference compared to pre-treatment $(p<0.05)$; \% variations are calculated from baseline.

12 months, had decreased by 33.7\% (Figure 2). At 6 months from the first injection, NSAID consumption decreased by $21.8 \%$, and at 12 months, had decreased by $41.7 \%$ (Figure 3 ). For both the Lequesne index and NSAID consumption, the reductions at 6 months compared to baseline, at 12 months compared to baseline, and at 12 months compared to 6 months were statistically significant ( $\mathrm{p}<0.05$ in all cases).

No systemic or severe local side effects were reported after the injection. In 5 out of 198 injections (2.5\%), there was a sensation of pain lasting from several hours to a few days that spontaneously regressed without requiring medical intervention.

\section{Discussion}

Several international scientific societies have developed recommendations concerning the use of VS in the form

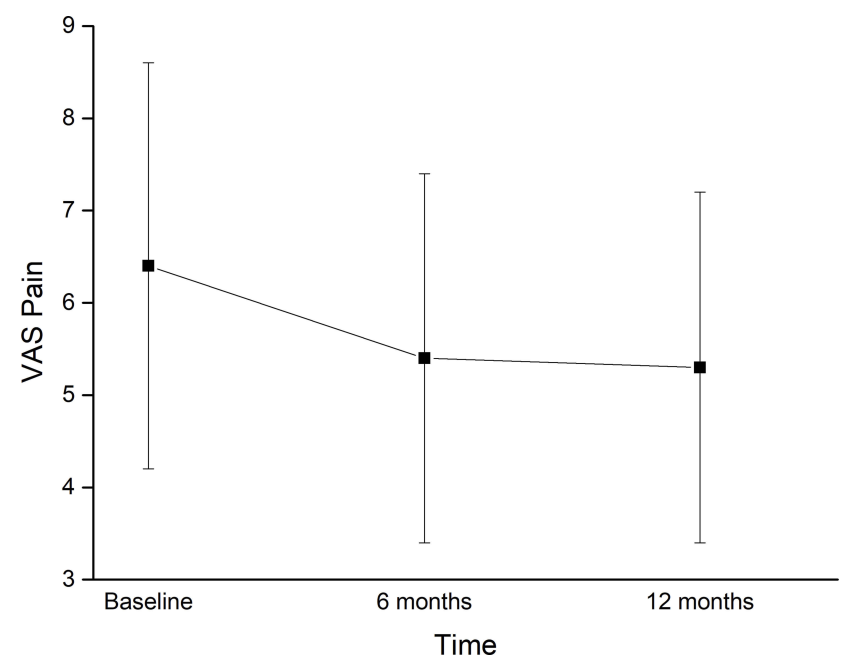

Figure I Decrease in pain over time, measured through a visual analogue scale (VAS). The decrease is statistically significant $(p<0.05)$ from baseline to 6 months and 12 months. 


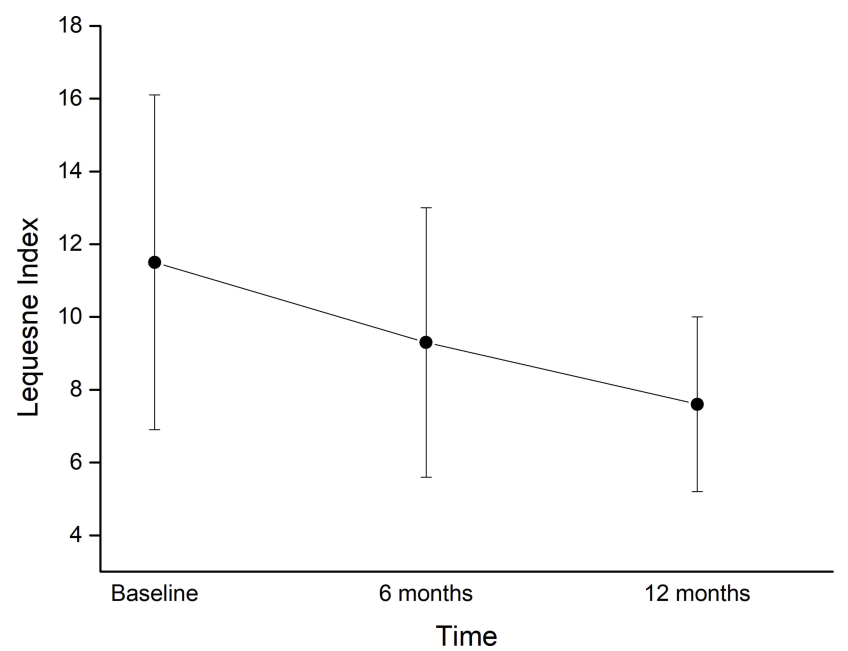

Figure 2 Decrease in Lequesne index over time. The decrease is statistically significant $(p<0.05)$ both from baseline to 6 and 12 months, and between 6 and 12 months.

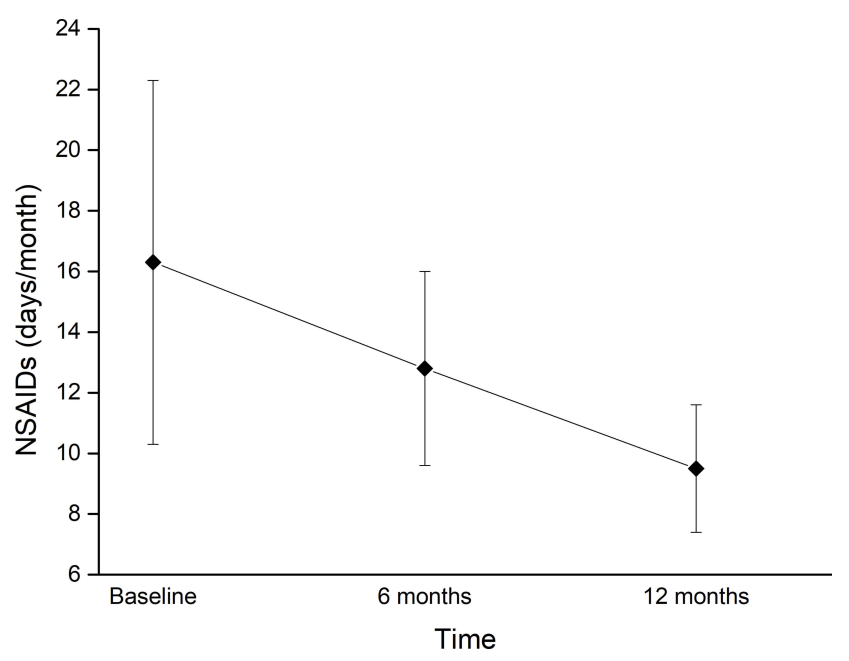

Figure 3 Decrease in NSAID consumption over time, measured in days/months. The decrease is statistically significant $(p<0.05)$ both from baseline to 6 and 12 months, and between 6 and 12 months.

of IA HA to manage symptoms of hip OA, indicating that it is a viable option as an adjuvant or, in less severe cases, an alternative to pharmacologic treatment. ${ }^{7,35}$ Yet, VS by HA injection is still under scrutiny by the scientific and clinical community, as it has been suggested that the data from controlled trials should be added with evidence from the real world setting and with longer follow-up. ${ }^{27}$

Furthermore, HA in different commercially available HA-based formulations may present different chemical modifications that have been introduced to improve the formulation rheology, resorption kinetics, and clinical effectiveness. Thus, one should assume that different HA-based formulations display different safety and effectiveness profiles, until proven otherwise. The matter is of clinical importance concerning safety and its consequences in clinical practice. The invasiveness of IA injections, in fact, may affect negatively the patient's adherence to the therapy course, resulting in the subject who rejects VS and relies once more only on medicine, and thus exposes him/herself to a greater risk of being affected by short- and long-term side effects. Thus, among HA formulations having equal effectiveness, one might prefer those involving a smaller number of IA injections. Less injections also implies a reduced risk for the patient, even when side effects of the formulations being compared are not different concerning their severity.

The results of the present study show that a single HYMOVIS ONE injection effectively managed symptoms of hip OA decreasing pain and reducing the need of NSAIDs intake over the 12 months of follow-up. The population of patients included had not been prospectively selected specifically to carry out this investigation and, even if reduced in size, may therefore be regarded as representative of the heterogeneous population that may be encountered, and treated, in common clinical practice.

The results of the present study are consistent with those observed by our group within the first year of treatment in a prospective, seven-year follow-up study where more than one IA injection of non-modified $(60 \mathrm{mg} / 4 \mathrm{~mL}) \mathrm{HA}^{32}$ was administered to 1022 hip OA patients to manage their OA symptoms. In this study, patients were categorized by age, gender, and body mass index (BMI), and the effect of IA injections was evaluated for each group by measuring how their Lequesne index, pain VAS, NSAID intake, and global medical and patient assessments varied over time. All groups showed a statistically significant reduction, at all time points compared to baseline, of all scores under assessment, with some differences concerning patients older than 70 years, whose scores were systematically higher at baseline than those of other age classes. VAS pain improvement at 1 year was lower $(-21 \%)$ in patients older than 70 and greater $(-39 \%)$ in other age classes. The decrease in the Lequesne index was similar in patients older than 70 $(-26 \%)$ and in other age classes $(-28 \%)$; NSAID consumption decreased in patients older than 70 years by $-27 \%$ and by $-33 \%$ in other age classes.

The results of the present study are also consistent with recent preliminary results by Rando et al ${ }^{36}$ concerning 30 subjects between 45 and 65 years of age who practiced tennis or amateur cycling for no less than 10 years and were affected by KL grade II or III hip OA. They were 
subjected to cycles of two IA US-guided HYMOVIS $(24 \mathrm{mg} / 3 \mathrm{~mL})$ injections 2 weeks apart from each other, repeated every 3 or 4 months, as required, during the 24 months of the study. All endpoints considered, including the Heidelberg Sports Activity Score (HAS) scale and the Copenhagen Hip and Groin Outcome Score (HAGOS) assessing symptoms, activity, limitations, participation restrictions, and quality of life, significantly improved over the study course, starting from the first IA injection.

The results of the present study are also comparable with those achieved using HYMOVIS to manage symptoms of knee OA patients. Priano et $\mathrm{al}^{25}$ analysed the retrospective data concerning 698 patients who received two infiltrations of $(24 \mathrm{mg} / 3 \mathrm{~mL})$ HYMOVIS, 1 week apart. The mean WOMAC scores were reduced by $56.3 \%$ from baseline. NSAID/acetaminophen use 2 times/week ( $48.8 \%$ of patients at baseline) was substantially reduced after 1 month and was $19.6 \%$ after 6 months. The effect on resting pain was rapid, strong, and lasting, as evident by a $56.8 \%$ reduction from baseline at 6 months and $53.6 \%$ at 12 months. Pain upon moving was reduced by $47.4 \%$ after 6 months and $46.0 \%$ after 12 months.

Although the results we have obtained are consistent with those just reported, they were achieved through a single HYMOVIS ONE injection.

The possibility that a reduced number of HYMOVIS injections may be as safe and effective as multiple ones has already been suggested by other authors. In 2010, Pavelka et $\mathrm{al}^{37}$ randomized 439 knee KL II or III stage OA patients either to 2 or 3 injections of $24 \mathrm{mg} / 3 \mathrm{~mL}$ HYMOVIS. Their results showed that there were no clinically relevant and/or statistically significant differences between the two treatment groups with regard to the primary outcome, that is, pain during a $50-\mathrm{ft}(15 \mathrm{~m})$ walk test, which was measured by VAS at all control visits, with the final reduction at 6 months being, in the two-injection group, $58.9 \%$.

Recently, Bernetti and Santilli ${ }^{38}$ showed that a single (32 mg/4 mL) HYMOVIS ONE IA injection is safe and effective in improving VAS, WOMAC, Knee injury and Osteoarthritis Outcome Score (KOOS), and biomechanical (gait analysis) scores over 12 months in 23 active patients. They emphasize how this result may be relevant because these patients, given their young age, may show low adherence to treatments involving multiple injections. The effectiveness of single HYMOVIS ONE IA injections to manage hip OA symptoms, as well as OA symptoms that affect other joints, should therefore be the subject of further, carefully designed investigations. The reasons that may be at the basis of the positive results that may be achieved through a single HYMOVIS ONE IA injection possibly consist in its enhanced rheological properties. The HYMOVIS HA molecule, in fact, consists of lateral hexadecyl (C-16) hydrophobic chains that facilitate its aggregation even at low aqueous concentrations, ${ }^{23}$ creating a mobile reticulum (MO.RE.). Because of the rheological properties of this formulation, it is an optimal viscosupplementation and shock absorbing agent. ${ }^{24}$

In a prospective, open-pilot study twelve patients affected by symptomatic hip OA underwent a single $2 \mathrm{~mL}$ Hylan G-F 20 (Synvisc, Sanofi Aventis - a high molecular weight, cross-linked hyaluronan derivative) ultrasoundguided intra-articular injection. Most subjects experienced a sustained improvement in their Lequesne score, the average reduction being $15 \%$ after 3 months. ${ }^{39}$ Pain reduction was statistically significant, compared to baseline, both 1 and 3 months after the injection, yet no significant difference was observed between the 1- and 3-month time points. A single Synvisc injection, therefore, did not provide a continuous improvement as it happened in the present study. ${ }^{39}$ The use of Hylan G-F 20 has been associated with a case of post-injection arthritis. ${ }^{40}$ The frequency and severity of adverse events observed in the present study were similar to those observed when analyzing data from the ANTIAGE registry related to 1906 patients who received 4002 injections with five different HA-based products according to our procedure, who experienced no systemic adverse events, and local pain reactions with a frequency ranging from $2.2 \%$ to $3.6 \%{ }^{33}$ Future studies with larger samples will have to confirm our data.

In a systematic review ${ }^{41}$ analysing 20 trials with a total of 3034 patients with knee osteoarthritis, the authors concluded that there was limited evidence showing a superior effect of Hylan G-F 20 compared to low molecular weight hyaluronic acids (LMWHA) in pain relief in the period from 2 to 3 months post injections. The authors speculated that this difference could be caused by a different resorption kinetic of the two formulations; accordingly, even HYMOVIS, given its molecular weight and characteristic rheology that may also affect its resorption kinetic, might perform better than LMWHA. Future controlled studies should be aimed to investigate if different formulations, including HYMOVIS ONE, Hylan G-F 20 and LMWHA, are equally safe and effective when administered according to a single injection protocol in prospectively recruited 
hip OA patients. Further studies should address the question if a single HYMOVIS injection is as effective as more injections, or if it is differently safe and effective than one or more injections of other HA-based formulations. Such studies would address one of the main limitations of the present study, that is the fact it lacks a control group.

Future studies should also address if single-injection protocols are more effective in specific subgroups of patients, when these are graded according to the most common scores used in clinical practice or according to other baseline characteristics, such as gender, BMI and OA severity. Also, HA IA single-injection protocols have never been studied over time periods longer than 12 months. Accordingly, further studies should be targeted at investigating the long-term response of hip OA patients when they undergo prolonged single-injection HYMOVIS ONE treatments.

\section{Conclusions}

A single HYMOVIS ONE injection was safe and effective for managing symptoms of patients affected by KellgrenLawrence grade I to III hip osteoarthritis, over a 12-month time period, in real-life conditions. Prospective studies with adequate sample size and design are needed to determine the criteria for the selection of patients that can benefit most by a single-injection protocol as well as to determine the optimal injection volume according to their characteristics and clinical signs at presentation.

\section{Disclosure}

Prof. Alberto Migliore acted as a consultant for and received grants from Pfizer, Abbvie, MSD, Fidia, Sanofi, and IBSA for national and international studies and courses. The authors report no other conflicts of interest in this work.

\section{References}

1. Zhang Y, Jordan JM. Epidemiology of osteoarthritis. Clin Geriatr Med. 2010;26(3):355-369. doi:10.1016/j.cger.2010.03.001

2. Roman-Blas JA, Bizzi E, Largo R, Migliore A, Herrero-Beaumont G. An update on the up and coming therapies to treat osteoarthritis, a multifaceted disease. Expert Opin Pharmacother. 2016;17 (13): 1745-1756. doi:10.1080/14656566.2016.1201070

3. Nemes S, Gordon M, Rogmark C, Rolfson O. Projections of total hip replacement in Sweden from 2013 to 2030. Acta Orthop. 2014;85 (3):238-243. doi:10.3109/17453674.2014.913224

4. Kurtz SM, Ong KL, Lau E, Bozic KJ. Impact of the economic downturn on total joint replacement demand in the United States: updated projections to 2021. J Bone Joint Surg Am. 2014;96(8):624-630. doi:10.2106/JBJS.M.00285
5. Bagge E, Bjelle A, Valkenburg HA, Svanborg A. Prevalence of radiographic osteoarthritis in two elderly European populations. Rheumatol Int. 1992;12(1):33-38. doi:10.1007/BF00246874

6. Sperati A, Picconi O, Tancioni V, Guasticchi G, Agabiti N. Outcomes of hip replacement: a hospital-based longitudinal study in Lazio region (Italy). Ann Ig. 2008;20(2):141-157.

7. Zhang W, Doherty M, Arden N, et al.; EULAR Standing Committee for International Clinical Studies Including Therapeutics (ESCISIT). EULAR evidence-based recommendations for the management of hip osteoarthritis: report of a task force of the EULAR Standing Committee for International Clinical Studies Including Therapeutics (ESCISIT). Ann Rheum Dis. 2005;64(5):669-681. doi:10.1136/ard.2004.028886.

8. Hochberg MC, Altman RD, April KT, et al.; American College of Rheumatology. American College of Rheumatology 2012 recommendations for the use of nonpharmacologic and pharmacologic therapies in osteoarthritis of the hand, hip, and knee. Arthritis Care Res (Hoboken). 2012;64(4):465-474. doi:10.1002/acr.21596.

9. Migliore A, Bizzi E, Herrero-Beaumont J, Petrella RJ, Raman R, Chevalier X. The discrepancy between recommendations and clinical practice for viscosupplementation in osteoarthritis: mind the gap!. Eur Rev Med Pharmacol Sci. 2015;19(7):1124-1129.

10. Henrotin Y, Raman R, Richette P, et al. Consensus statement on viscosupplementation with hyaluronic acid for the management of osteoarthritis. Semin Arthritis Rheum. 2015;45(2):140-149. doi:10.10 16/j.semarthrit.2015.04.011

11. Paoloni M, Bernetti A, Belelli A, et al. Appropriateness of clinical and organizational criteria for intra-articular injection therapies in osteoarthritis. A Delphi method consensus initiative among experts in Italy. Ann Ist Super Sanita. 2015;51(2):131-138. doi:10.4415/ ANN_15_02_11

12. Qvistgaard E, Kristoffersen H, Terslev L, Danneskiold-Samsøe B, Torp-Pedersen S, Bliddal H. Guidance by ultrasound of intra-articular injections in the knee and hip joints. Osteoarthr Cartil. 2001;9: 512-517. doi:10.1053/joca.2001.0433

13. Migliore A, Martin LS, Alimonti A, Valente C, Tormenta S. Efficacy and safety of viscosupplementation by ultrasound-guided intraarticular injection in osteoarthritis of the hip. Osteoarthr Cartil. 2003;11:305-306. doi:10.1016/S1063-4584(03)00008-6

14. Fraser JR, Laurent TC, Laurent UB. Hyaluronan: its nature, distribution, functions and turnover. $J$ Intern Med. 1997;242(1):27-33. doi:10.1046/j.1365-2796.1997.00170.x

15. Band PA, Heeter J, Wisniewski HG, et al. Hyaluronan molecular weight distribution is associated with the risk of knee osteoarthritis progression. Osteoarthr Cartil. 2015;23(1):70-76. doi:10.1016/j. joca.2014.09.017

16. Balazs EA, Watson D, Duff IF, Roseman S. Hyaluronic acid in synovial fluid. I. Molecular parameters of hyaluronic acid in normal and arthritis human fluids. Arthritis Rheum. 1967;10(4):357-376. doi:10.1002/art.1780100407

17. Balazs EA, Denlinger JL. Sodium hyaluronate and joint function. Equine Vet Sci. 1985;5:217-228. doi:10.1016/S0737-0806(85)80102-7

18. Bothner H, Wik O. Rheology of hyaluronate. Acta Otolaryngol Suppl. 1987;442:25-30. doi:10.3109/00016488709102834

19. Cullis-Hill D, Ghosh P. The role of hyaluronic acid in joint stability-a hypothesis for hip dysplasia and allied disorders. Med Hypotheses. 1987;23(2):171-185. doi:10.1016/0306-9877(87)90153-8

20. Dahl LB, Dahl IM, Engström-Laurent A, Granath K. Concentration and molecular weight of sodium hyaluronate in synovial fluid from patients with rheumatoid arthritis and other arthropathies. Ann Rheum Dis. 1985;44(12):817-822. doi:10.1136/ard.44.12.817

21. Collins MN, Birkinshaw C. Physical properties of crosslinked hyaluronic acid hydrogels. J Mater Sci Mater Med. 2008;19 (11):3335-3343. doi:10.1007/s10856-008-3476-4

22. Highley CB, Prestwich GD, Burdick JA. Recent advances in hyaluronic acid hydrogels for biomedical applications. Curr Opin Biotechnol. 2016;40:35-40. doi:10.1016/j.copbio.2016.02.008 
23. Finelli I, Chiessi E, Galesso D, Renier D, Paradossi G. Gel-like structure of a hexadecyl derivative of hyaluronic acid for the treatment of osteoarthritis. Macromol Biosci. 2009;9(7):646-653. doi:10.1002/mabi.v9:7

24. Finelli I, Chiessi E, Galesso D, Renier D, Paradossi G. A new viscosupplement based on partially hydrophobic hyaluronic acid: a comparative study. Biorheology. 2011;48(5):263-275. doi:10.323 3/BIR-2011-0596

25. Priano F. Early Efficacy of Intra-Articular HYADD ${ }^{\circledR} 4$ (Hymovis $^{\circledR}$ ) Injections for Symptomatic Knee Osteoarthritis. Joints. 2017;5 (2):79-84. doi:10.1055/s-0037-1603677.

26. Bisicchia S, Bernardi G, Tudisco C. HYADD 4 versus methylprednisolone acetate in symptomatic knee osteoarthritis: a single-centre single blind prospective randomised controlled clinical study with 1-year follow-up. Clin Exp Rheumatol. 2016;34(5):857-863.

27. Migliore A, Tormenta S, Massafra U, et al. Repeated ultrasound-guided intra-articular injections of $40 \mathrm{mg}$ of Hyalgan may be useful in symptomatic relief of hip osteoarthritis. Osteoarthr Cartil. 2005;13(12):1126-1127. doi:10.1016/j.joca.2005. 08.001

28. Migliore A, Bizzi E, Massafra U, Alimonti A, Martin S, Tormenta S. 18 months follow-up after intra-articular administration of Hyalubrix ${ }^{\circledR}$ in 344 patients with symptomatic osteoarthritis of the hip. Osteoarth Cartil. 2008;16(S4):S118-S119. doi:10.1016/S10634584(08)60306-4

29. Migliore A, Tormenta S, Massafra U, et al. Intra-articular administration of hylan G-F 20 in patients with symptomatic hip osteoarthritis: tolerability and effectiveness in a large cohort study in clinical practice. Curr Med Res Opin. 2008;24(5):1309-1316. doi:10.1185/ 030079908X291930

30. Migliore A, Massafra U, Bizzi E, et al. Comparative, double-blind, controlled study of intra-articular hyaluronic acid (Hyalubrix) injections versus local anesthetic in osteoarthritis of the hip. Arthritis Res Ther. 2009;11(6):R183. doi:10.1186/ar2875

31. Migliore A, Massafra U, Bizzi E, et al. Intra-articular injection of hyaluronic acid (MW 1,500-2,000 kDa; HyalOne) in symptomatic osteoarthritis of the hip: a prospective cohort study. Arch Orthop Trauma Surg. 2011;131(12):1677-1685. doi:10.1007/s00402-0111353-y

32. Migliore A, Massafra U, Frediani B, et al. HyalOne ${ }^{\circledR}$ in the treatment of symptomatic hip OA - data from the ANTIAGE register: seven years of observation. Eur Rev Med Pharmacol Sci. 2017;21 (7):1635-1644.
33. Migliore A, Tormenta S, Laganà B, et al. Safety of intra-articular hip injection of hyaluronic acid products by ultrasound guidance: an open study from ANTIAGE register. Eur Rev Med Pharmacol Sci. 2013;17 (13):1752-1759

34. Kellgren JH, Lawrence JS. Radiological assessment of osteo-arthrosis. Ann Rheum Dis. 1957;16(4):494-502. doi:10.1136/ard.16.4.494

35. Bannuru RR, Osani MC, Vaysbrot EE, et al. OARSI Guidelines for the Non-Surgical Management of Knee, Hip, and Polyarticular Osteoarthritis. Osteoarthr Cartil. 2019;27(11):S1063-4584(19)31116-1.

36. Rando G, Pastorino R, Chiacchio F, Franzé F. Valutazione degli effetti a lungo termine del trattamento intrarticolare dell'anca con un acido ialuronico reticolare in sportivi affetti da coxartrosi (Long-term effects of intra-articular hip treatment using a reticular hyaluronic acid in sportsmen affected by hip osteoarthritis). Oral communication to the 7th National ANTIAGE Symposium; October 2018; Rome, Italy.

37. Pavelka K, Niethard FU, Giordan N. A multicentre, international, double blind, randomized, placebo - controlled study to assess the efficacy and safety of 2 different regimens of HYADD4-G in knee osteoarthritis. Osteoarthr Cartil. 2010;18(Supplement 2):S45-S256. doi:10.1016/S1063-4584(10)60353-6

38. Bernetti A, Santilli V. Prospective, Monocentric, Post-CE Mark Study to Assess Efficacy and Safety of Hymovis One (32 Mg/4 Ml) IntraArticular Injection in Active Patients Affected by Knee Overuse Syndrome. Abstract to the World Congress on Osteoporosis, Osteoarthritis and Musculoskeletal Diseases (WCO-IOF-ESCEO). Rome: Abstract Book; April 2019:342-343.Available from: https:// www.wco-iof-esceo.org/sites/wco19/pdf/WCO19-AbstractBook.pdf. Accessed October 19, 2019.

39. Migliore A, Tormenta S, Martin LS, et al. Open pilot study of ultrasound-guided intra-articular injection of hylan G-F 20 (Synvisc) in the treatment of symptomatic hip osteoarthritis. Clin Rheumatol. 2005;24(3):285-289. doi:10.1007/s10067-004-1009-1

40. Kocak D, Annaswamy T, Chong S, Arora R. Development of systemic inflammatory polyarthritis after zygapophyseal joint injection with hylan G-F 20 (synvisc-one). Am J Phys Med Rehabil. 2018;97 (2):147-149. doi:10.1097/PHM.0000000000000797

41. Zhao H, Liu H, Liang X, Li Y, Wang J, Liu C. Hylan G-F 20 versus low molecular weight hyaluronic acids for knee osteoarthritis: a meta-analysis. BioDrugs. 2016;30(5):387-396. doi:10.1007/s40259016-0186-1
Orthopedic Research and Reviews

\section{Publish your work in this journal}

Orthopedic Research and Reviews is an international, peer-reviewed, open access journal that focusing on the patho-physiology of the musculoskeletal system, trauma, surgery and other corrective interventions to restore mobility and function. Advances in new technologies, materials, techniques and pharmacological agents are particularly welcome. The manuscript management system is completely online and includes a very quick and fair peer-review system, which is all easy to use. Visit http://www.dovepress.com/testimonials.php to read real quotes from published authors. 